\title{
Estrategia Nacional sobre Drogas
}

\author{
Robles, G.
}

Delegado del Gobierno para el Plan Nacional sobre Drogas

\section{RESUMEN}

El pasado 17 de diciembre de 1999 se aprobó por Real Decreto la Estrategia Nacional sobre Drogas 2000-2008, fruto del consenso y las aportaciones de las distintas instituciones y organizaciones que conforman el Plan Nacional sobre Drogas. En ella se determinan, a partir de un análisis previo de la situación, las principales metas y objetivos a conseguir en la próxima década en las diferentes áreas de intervención: prevención, asistencia, reducción de daños, asistencia, integración social, investigación y formación. La estrategia se basa fundamentalmente en la prevención de los consumos y sus consecuencias, con especial atención al tabaco, alcohol y los consumos recreativos de drogas, de conformidad con el trabajo desarrollado en el Plan Nacional a lo largo de los últimos años. Se hacen también propuestas de evaluación para hacer un seguimiento del desarrollo de la estrategia.

Palabras clave: estrategias preventivas, políticas nacionales, España.

\section{ABSTRACT}

The Royal Decree on the National Drugs Strategy approved on 17th December 1999, is the result of the consensus and contributions of the various institutions and organisations comprising the National Drug Plan. On the basis of a prior analysis of the situation, it determines the principal goals and objectives to be attained during the next decade in the various areas of intervention, prevention, assistance, risk reduction, social integration, research and training. The strategy is fundamentally based on the prevention of drug use and its consequences, with particular emphasis on tobacco, alcohol and recreational drug use in accordance with the work developed in the National Plan over the last few years. It also puts forward proposals for the evaluation and follow-up of the outcome of the strategy.

Key words: preventive strategies, policy, Spain.
$\mathbf{L}$ a Estrategia Nacional sobre Drogas, que se configura como un auténtico nuevo Plan Nacional, adaptado a las circunstancias actuales y a los retos del futuro, fue aprobada por el Gobierno mediante Real Decreto el pasado 17 de diciembre de 1999.

Las razones que justifican la elaboración de la Estrategia son básicamente dos: a) Por un lado, tras quince años de vigencia, el Plan Nacional sobre Drogas necesitaba adecuarse a la realidad siempre cambiante de las drogodependencias, sobre todo dadas las transformaciones que ha experimentado este fenómeno en los últimos años y b) Por otra parte, los compromisos internacionales adquiridos por España. Así, la XX Sesión Extraordinaria de la Asamblea General de Naciones Unidas, celebrada en junio de 1998, aprobó una declaración en la que se pedía a todos los Estados la elaboración de estrategias nacionales para conseguir una serie de objetivos centrados en dos fechas: los años 2003 y 2008.
De acuerdo con todo ello, los responsables del Plan Nacional sobre Drogas acordamos recoger en un documento, de forma clara y precisa, las metas y objetivos a alcanzar en esas fechas en las diferentes áreas de intervención del Plan.

Entre las áreas de intervención que comprende la Estrategia cabe señalar la prevención del consumo de drogas, la reducción de los daños y la asistencia e integración social. Otras áreas también destacadas son el control de la oferta, la cooperación internacional, la regulación normativa, la investigación y formación, la evaluación y los sistemas de información y la financiación.

\section{PRINCIPALES OBJETIVOS A CONSEGUIR EN LA PREVENCIÓN DEL CONSUMO}

La Estrategia Nacional sobre Drogas se fundamenta prioritariamente en la prevención de los consumos 
y sus consecuencias, con especial atención al alcohol, el tabaco y los consumos de carácter recreativo, siendo todo ello coherente con el trabajo iniciado durante los últimos años.

Entre las grandes metas que se plantean en materia de prevención, cabe destacar:

1. Ofrecer a la población información suficiente sobre los riesgos del consumo de tabaco, alcohol y las otras sustancias que pueden general dependencia, así como poner en marcha medidas de control sobre la publicidad y promoción de bebidas alcohólicas y tabaco que protejan a los menores de edad.

2. Potenciar la formación del personal docente de educación primaria y secundaria sobre problemas relacionados con el consumo de drogas.

3. Lograr que la mayoría de los escolares, al finalizar la educación obligatoria hayan recibido información objetiva suficiente y formación adecuada sobre las consecuencias del uso y abuso de las drogas y adquirido habilidades suficientes para abordar su relación con las mismas.

Por lo tanto, la Estrategia propone una generalización progresiva de la prevención escolar.

4. Estimular e implementar estrategias preventivas en la población laboral.

\section{PRINCIPALES OBJETIVOS A CONSEGUIR EN LA REDUCCIÓN DE LOS DAÑOS}

La Estrategia contempla el incremento y mejora de aquellas intervenciones dirigidas a reducir los daños y riesgos ocasionados por los consumos de drogas, fundamentalmente en aspectos sociales y de salud. En este sentido cabe señalar como objetivos más destacados:

1. Procurar que la mayor parte de la población drogodependiente pueda acceder a programas de reducción de daños, especialmente los programas de intercambio de jeringuillas, sexo más seguro y consumo de menos riesgo.

2. Implantar programas de educación sanitaria tendentes a la reducción del daño por consumo de alcohol, tabaco y otras drogas. Especialmente, se impulsarán estos programas asociados al consumo de alcohol en relación con los accidentes de tráfico y la violencia.

3. Impulsar la calidad de los programas de reducción de daños y su coordinación funcional con la red asistencial del Sistema Nacional de Salud.

4. Diversificar la oferta de los programas de reducción de daños en los centros penitenciarios mediante iniciativas tales como la extensión de los programas de intercambio de jeringuillas.

\section{PRINCIPALES OBJETIVOS A CONSEGUIR EN LA ASISTENCIA E INTEGRACIÓN SOCIAL}

Una de las principales apuestas de la Estrategia consiste en garantizar la plena asistencia ambulatoria a los drogodependientes en el ámbito de su Comunidad o Ciudad Autónoma y dentro del Área de Salud de su residencia.

Igualmente contempla que, cumplidas las necesidades de cobertura universal asistencial, se preste atención a las desigualdades y muy especialmente a aquellos colectivos de drogodependientes que no contacten con las redes asistenciales. mos:

Entre los objetivos más importantes, destacaría-

1. Mejorar el abordaje terapéutico de las personas que tienen problemas con el consumo del alcohol y el tabaco, las nuevas drogas y los nuevos patrones de consumo.

2. Impulsar los programas de detección precoz e intervención temprana dirigidos a menores con problemas de consumo de drogas y a los hijos de adictos.

3. Garantizar la atención a las personas detenidas y/o ingresadas en prisión con problemas relacionados con el consumo de drogas.

4. Promover la integración laboral de los drogodependientes, facilitando su incorporación a la sociedad en calidad de miembros activos de la misma.

A estos efectos, los Planes Autonómicos sobre Drogas y los municipios de más de 20.000 habitantes tendrán programas de formación y empleo normalizados o específicos para drogodependientes.

\section{PRINCIPALES OBJETIVOS A CONSEGUIR EN INVESTIGACIÓNY FORMACIÓN}

En cuanto a la investigación, la Delegación del Gobierno para el PNSD, actuará en dos vertientes: consolidando la investigación sobre drogodependencias a nivel estatal, por un lado, e impulsando e incentivando la investigación aplicada en las Comunidades y Ciudades Autónomas.

La formación, por su parte, debe entenderse como un proceso continuo, con tres niveles básicos: formación de pregrado, formación de postgrado universita- 
rio y formación continuada para profesionales de las drogodependencias y de los servicios generales. Esta última dimensión afecta de modo muy directo a un colectivo como el que $\mathrm{Vds}$. representan.

Como objetivos a conseguir, hay que señalar:

1. Con carácter general, garantizar la formación básica, especializada y continuada de los universitarios y profesionales, así como el acceso a la documentación y creación y desarrollo de centros y equipos de investigación aplicada en materia de drogodependencias.

2. Creación de un Instituto Nacional de Investigación y Formación sobre Drogas, dependiente de esta Delegación del Gobierno, con funciones de impulso y mejora de la calidad de los programas en esta materia.

3. Puesta en marcha de una red autonómica de puntos focales de documentación, con la utilización de nuevas tecnologías de acceso a la información.
PRINCIPALES OBJETIVOS A CONSEGUIR EN EVA-
LUACIÓNY SISTEMAS DE INFORMACIÓN

Finalmente, la Estrategia prevé el diseño de un sistema de evaluación que permita la detección precoz de las desviaciones en los objetivos marcados, a fin de generar las acciones correctivas pertinentes. Por otro lado, la puesta en marcha del Observatorio Español sobre Drogas ha supuesto la existencia de un organismo clave a la hora de contar con información fidedigna y actualizada sobre el fenómeno de las drogodependencias y poder obrar en consecuencia.

Nuestro principal objetivo es evitar que las generaciones más jóvenes se sientan atraídas por el consumo de sustancias, como el alcohol y otras drogas, creyendo que de esa forma pueden experimentar sensaciones que les hagan más interesante la vida. De ahí nuestra incidencia en la prevención y en la promoción de valores y estilos de vida incompatibles con ese consumo, al tiempo que no podemos escatimar una respuesta asistencial profesionalizada y eficaz a todas aquellas personas que han sucumbido a la adicción a las drogas. 
